\title{
The effect of problem size on representation in deductive problem solving*
}

\author{
JOHN M. POLICH \\ Wayne State University, Detroit, Michigan 48202 \\ and \\ STEVEN H. SCHWARTZ \\ University of Massachusetts-Boston, Boston, Massachusetts 02116
}

\begin{abstract}
Adult Ss attempted to solve logical deductive reasoning problems that varied systematically in amount of information presented. Methods Ss employed in representing the problem were classified into five main types or modes. The proportion of Ss using a matrix mode of problem representation increased moderately with problems containing large amounts of information. The performance of Ss using a matrix mode of representation suggested that this advantage is related to ease of applying and/or storing the results of logical operations in such a problem space rather than to any facilitative effects of encoding processes. Specific limits to normal processing are hypothesized.
\end{abstract}

A major component in a problem-solving task is the encoding of the problem elements and their interrelationships into a particular representation or space. This process serves to isolate the elements pertinent to solution and delimits a set of relevant operations that directs transformations of these elements (Newell, Shaw, \& Simon, 1958; Greeno, 1973). Hence, a central task for investigators of human problem solving has been to define the constituent elements of a solver's problem space and the types or modes of representation employed in various situations.

Schwartz (1971) reported a study that explored some modes of representation that Ss employed when at tempting to solve several "who-done-it" type deductive reasoning problems. An example of this type of problem is presented in Table 1. The elements of these problems varied systematically on a number of dimensions or categories (e.g., spy's name, contact location, specialty, etc.), with each dimension having a number of attributes or values (e.g., Boris, Edmond, George, Irving; Peking, London, Tokyo, Paris; etc.).

The Ss were presented a series of such problems and were encouraged to show all work as they attempted to solve them. The resulting protocols were then classified into five categories according to the manner in which the $S$ represented or structured the problem information: (1) a matrix format, (2) a more general grouping mode, (3) a network or graphic representation, (4) a rewriting of the problem in sentence form, or (5) a miscellaneous category.

Schwartz (1971) found a striking relationship between mode of representation and solution

*Portions of this paper were presented at the meeting of the Psychonomic Society, St. Louis, November 1972. The authors wish to thank George Potts for his critical comments on an early draft of this paper. Requests for reprints should be sent to John M. Polich, Department of Psychology, Dartmouth College, Hanover, New Hampshire 03755. performance. Though only about $25 \%$ of the Ss employed a matrix format for representing the problem information, $75 \%$ of those doing so correctly solved the problems. This compared with solution rates varying from $25 \%$ to $50 \%$ for Ss using the other categories of problem representation. It was suggested that the effect of particular task variables on solution performance was largely a function of the degree to which they influenced the mode of representation that Ss used in structuring the problem information.

The present study was designed to extend this hypothesis by investigating the influence of information quantity on representation type and solution performance. The amount of information in these tasks can be controlled systematically by varying the number of dimensions and values, as well as by varying the

Table 1

Example of Deductive Reasoning Problem (Four Dimensions, Four Values)

This is a problem about four different spies. Each of the spies has his contact located in a different location. Each spy also has a different spy specialty and a different color of hair.

1. The spy with brown hair specializes in secret missile plans.

2. The spy whose contact is located in Peking specializes in germ warfare plans.

3. One of the spies has red hair.

4. The spy who specializes in electronic bugs has his contact located in Tokyo.

5. Paris is the contact location of one of the spies.

6. The spy who has black hair has his contact located in London.

7. The spy named Irving has gray hair.

8. One of the spies is named Boris.

9. The spy whose contact is located in London is named Edmond.

10. The spy named George specializes in germ warfare. 11. One of the spies specializes in scientific papers.

What is the name of the spy whose contact is in Tokyo? 
redundancy of information presented in the problem statements. Previous studies have demonstrated that use of the matrix mode of representation results in fewer deductive errors (Schwartz, 1971). It is reasonable to argue that, in an attempt to minimize the possibility of logical errors, use of the matrix mode of representation will increase with increases in problem information. Though use of the matrix mode reduces logical errors, its use does not necessarily assure a correct solution, since a considerable number of errors have been found to occur during the transformation of originally presented information into a matrix representation (Schwartz \& Fattellah, 1972).

\section{METHOD}

\section{Subjects}

A total of 180 Ss were randomly selected from the introductory psychology course at a large urban university. Each $\mathrm{S}$ participated in one $50 \mathrm{~min}$ session to fulfill a course requirement.

\section{Materials}

The task problems consisted of nine logical deductive reasoning problems modeled after those reported by Schwartz (1971). The problems were constructed with either three, five, or seven dimensions and three, five, or seven values, with the same elements of the 7 by 7 problem used in generating the smaller problems. All of the experimental problem statements were stated affirmatively; the information in each problem was limited to the minimum number of statements required to produce a correct solution. The problems were arranged so that they necessitated a complete or nearly complete correct compilation of all the problem relations in order to finally arrive at the correct solution.

The problems were presented in sentence form as a paper and pencil task with several blank sheets of paper included in the task booklet for the $S$ to note his method of solution. A second problem consisting of seven dimensions and seven values was also provided as an additional time filler to prevent disruption of the group due to early solvers leaving.

\section{Design}

A 3 by 3 factorial design was employed which varied the number of dimensions (three, five, seven) and the number of values (three, five, seven) used in constructing a problem for each cell. The Ss were randomly assigned to each experimental condition (20 Ss per cell) with an equal proportion of males and females within a cell. The order of the presentation of the various sizes of task problems was block randomized over the nine problems. Thus, within a given experimental session, some Ss received the smaller problems (e.g., 3 by 3 ) while others recsived the larger problems (e.g., 7 by 7 ), with all $S$ s required to attempt a solution for the filler problem.

\section{Procedure}

The problems were presented to groups of about $10 \mathrm{Ss}$ in a large classroom. After passing out the test booklets, the E read the instructions on the front page of the booklet which explained that the experiment concerned problem solving and that the Ss were to attempt to solve the problems presented to them. The $\mathrm{E}$ emphasized the desirability of a good attempt to solve the problem, despite its possible difficulty, several times throughout the reading of the instructions. The Ss were further instructed and greatly encouraged to show all of their work on the problems in order that the $\mathrm{E}$ might be able to judge how the $S$ went about solving the problem. These instructions had previously demonstrated excellent results in obtaining classifiable modes of representation.
The Ss were asked to indicate when the first experimental problem had been completed; the $\mathrm{E}$ then examined their solution attempt. If the $\mathbf{S}$ had complied with the minimum amount of effort required (i.e., had generated some sort of problem representation and not obviously guessed), he was given the filler problem and told to proceed. The $E$ then noted the time in minutes from the beginning of the experimental session to the time signaled for the solution attained. All Ss were thus required to work the entire 45-min session despite the fact that some received problems of a smaller size and were able to finish more quickly than those Ss who initially received problems of a larger structure. Those Ss who did not complete a solution for the experimental problem received a time score of $45 \mathrm{~min}$.

\section{RESULTS}

A protocol was classified as solved if the $S$ had correctly answered the problem question directly or if he had generated a representation of the problem information which indicated the correct solution. If the relevant answer was obviously incorrect or ambiguously placed within the problem representation, that $S$ was judged as a nonsolver. Problem representations were independently classified by three judges using the categories reported by Schwartz (1971). All protocols were characterized as a matrix, local grouping, network, sentence rewrite, or miscellaneous representation, with an intraclass correlation as an estimate of reliability between judges computed at $r=98$. Differences in the classification of a specific S's protocol were resolved by a majority opinion. Only the experimental problems were classified.

The proportion of Ss using each type of representation, the corresponding solution rates, and mean time to solution (in minutes) averaged over experimental conditions are presented in Table 2 . The matrix mode of representation was used most often across all experimental conditions (44\%), with the local grouping category obtaining the next highest proportion of use $(37 \%)$, and the other three categories receiving relatively little use. Averaging over experimental conditions, Ss who employed the matrix solved a greater proportion of the problems (62\%) than did Ss who used a local grouping representation (44\%). This difference was significant, $\mathrm{z}=2.17, \mathrm{p}<.02$, using a binomial test with pooled sample variances. Even more dramatically, note that on the three most difficult problems ( 5 by 7,7 by 5 , and 7 by 7 ), not a single $S$ solved who had not represented the problem in a matrix format.

The need for a matrix mode of representation in larger problems was recognized by some Ss, particularly as the number of values increased from three to five to seven, as indicated by the analysis of variance on proportion of matrix use across problem values, $F(2,171)=2.89, p<.06$. In general, however, Ss were not as sensitive as we had expected to the possible advantages of a matrix mode of representation with problems of a greater number of dimensions. The increase in matrix use across dimensions was even weaker, $F(2,171)=1.73, p<.20$, with the $\mathrm{SE}=.11$ for both $F$ ratios. The possibility that the use of a matrix 
Table 2

Proportion of Times Each Mode of Representation Was Used, Corresponding Solution Rates, and Mean Time to Solution Data for Each Experimental Condition $(N=20)$

\begin{tabular}{|c|c|c|c|c|c|c|c|c|c|c|c|c|c|}
\hline \multirow{4}{*}{$\begin{array}{l}\text { Problem } \\
\text { Size } \\
\text { D by V }\end{array}$} & \multicolumn{10}{|c|}{ Representation Used } & \multirow{4}{*}{$\begin{array}{l}\text { Total } \\
\text { Pro- } \\
\text { portion } \\
\text { Solving }\end{array}$} & \multirow{3}{*}{\multicolumn{2}{|c|}{ Time to Solution }} \\
\hline & \multicolumn{2}{|c|}{ Matrix } & \multicolumn{2}{|c|}{ Grouping } & \multicolumn{2}{|c|}{ Network } & \multicolumn{2}{|c|}{ Rewrite } & \multicolumn{2}{|c|}{ Miscellaneous } & & & \\
\hline & & Solu- & & Solu- & & Solu- & & Solu- & & Solu- & & & \\
\hline & Use & tion & Use & tion & Use & tion & Use & tion & Use & tion & & & SE* \\
\hline 3 by 3 & .40 & .88 & .35 & .86 & .05 & 1.00 & .15 & .07 & .05 & .00 & .80 & 12.80 & 1.34 \\
\hline 3 by 5 & .40 & .88 & .35 & .86 & .00 & - & .10 & 1.00 & .15 & .00 & .75 & 16.25 & 1.58 \\
\hline 3 by 7 & .50 & 1.00 & .30 & .50 & .00 & - & .15 & 1.00 & .05 & .00 & .80 & 21.15 & 2.44 \\
\hline 5 by 3 & .25 & 1.00 & .35 & .71 & .10 & .00 & .25 & .60 & .05 & .00 & .65 & 21.80 & 2.36 \\
\hline 5 by 5 & .35 & .43 & .45 & .33 & .10 & .00 & .10 & .00 & .00 & - & .30 & 33.35 & 2.49 \\
\hline 5 by 7 & .50 & .20 & .45 & .00 & .05 & .00 & .00 & - & .00 & - & .10 & 43.05 & 0.94 \\
\hline 7 by 3 & .35 & 1.00 & .35 & .71 & .05 & .00 & .10 & .50 & .15 & .33 & .70 & 26.05 & 2.44 \\
\hline 7 by 5 & .60 & .08 & .40 & .00 & .00 & - & .00 & - & .00 & - & .05 & 39.55 & 1.80 \\
\hline 7 by 7 & .65 & .08 & .30 & .00 & .05 & .00 & .00 & - & .00 & - & .05 & 38.90 & 2.24 \\
\hline Mean & .44 & .62 & .37 & .44 & .04 & .11 & .09 & .42 & .05 & .04 & .47 & 28.10 & 1.98 \\
\hline
\end{tabular}

*Standard error of the mean for time to solution scores

representation is indicative of overall higher intelligence which then moderates successful performance received little support as correlations between Standard Aptitude Test (SAT) scores and either performance or matrix use were insignificant $(r=.08$ and .11 , respectively).

Averaging over mode of representation, the proportion of $\mathrm{Ss}$ correctly solving the problems decreased with increases in both the number of dimensions, $F(2,171)=29.48, p<.001$, and the number of values, $F(2,171)=18.19, \mathrm{p}<.001$. Problems which had only three dimensions were solved with about the same frequency no matter how many values were involved, but when the number of dimensions was increased to five or seven, a sharp decrement in solution rate was observed. This interaction was significant, $\mathrm{F}(4,171)=4.83, \mathrm{p}<.001$, with the $\mathrm{SE}=.09$ for all three $F$ ratios. Time required to solve showed similar trends, increasing with both the number of dimensions, $\mathrm{F}(2,171)=75.01, \mathrm{p}<.001$, and values, $\mathrm{F}(2,171)=$ $39.81, p<.001$. The interaction between these factors inversely mimicked the trends produced by the proportion of Ss solving under each experimental condition and likewise was significant, $F(4,171)=4.17$, $\mathrm{p}<.003, \mathrm{SE}=1.98$ for each of these analyses. Since time to solution frequently produces skewed distributions, the analysis of variance was also performed on the square root transformation of the time scores. No appreciable difference in the resulting $F$ ratios was observed.

The protocols were analyzed further for specific types of errors in the representation of the problem information. Each S's representation was translated into a standard matrix format for the particular dimension by value size of the problem. From this standard representation two types of relationships between the problem elements were distinguished: (1) explicit or given problem relations -information about the relationship of problem elements which were overtly presented in the problem statements that the $S$ read; (2) implicit or deduced problem relations-information about problem relationships which was not directly stated, but which demanded an inference from the presented information. For example, in the sample deductive problem presented in Table 1 , an explicit relation is contained in Sentences 6 and 9. The spy with black hair with his contact located in London is given as well as the spy Edmond and the contact location of London. Thus, the relation between elements Edmond and black hair is effectively made explicit by the problem statements. An implicit problem relation is suggested by Sentences 4 and 7. The spy who specializes in electronic bugs has his contact in Tokyo, and the spy named Irving is known to have gray hair. Further analysis reveals, however, that Irving is also the only spy who can possibly have his contact located in Tokyo because of the restrictions generated by the other problem relations (i.e., all other options are determined by the remaining problem statements). Thus, the $S$ must infer the relationship between these problem elements by logically deducing that it is the only possible means of accounting for these elements.

Specific types of errors in representation of problem information were counted for both of these types of relationships. The proportion of those relations which were left out all together from the S's representation was computed as the omission error rate. The proportion of those relations which were incorrectly positioned or associated in the S's representation was computed as the commission error rate. A summary of the mean proportion of representation errors for each type of representation and problem relation-error category combination is presented in Table 3.

Averaged over all experimental conditions and modes of representation, Ss made a large number of errors on implicit problem relations (56\%) and very few errors on explicit relations $(11 \%)$. This difference was significant, $\mathrm{z}=9.10, \mathrm{p}<.001$. Since the proportion of errors made with explicit relationships is comparatively small across all modes of representation, it is not surprising that mode of representation had little effect for these 
Table 3

Mean Proportion of Representation Errors

\begin{tabular}{|c|c|c|c|c|c|c|c|}
\hline \multirow{2}{*}{\multicolumn{2}{|c|}{ Type of Error }} & \multicolumn{5}{|c|}{ Representation } & \multirow[b]{2}{*}{$\begin{array}{c}\text { Mean } \\
\mathrm{N}=180\end{array}$} \\
\hline & & $\begin{array}{l}\text { Matrix } \\
N=80\end{array}$ & $\begin{array}{c}\text { Grouping } \\
\mathrm{N}=66\end{array}$ & $\begin{array}{c}\text { Network } \\
\mathrm{N}=8\end{array}$ & $\begin{array}{c}\text { Rewrite } \\
\mathrm{N}=17\end{array}$ & $\begin{array}{c}\text { Miscellaneous } \\
\mathrm{N}=9\end{array}$ & \\
\hline \multirow{3}{*}{ Explicit } & Omission & .04 & .06 & .21 & .04 & .64 & .08 \\
\hline & Commission & .02 & .02 & .03 & .07 & .00 & .03 \\
\hline & Total & .06 & .08 & .24 & .12 & .64 & .11 \\
\hline \multirow{4}{*}{ Implicit } & Omission & .32 & .54 & .67 & .46 & .85 & .46 \\
\hline & Commission & .09 & .10 & .12 & .17 & .10 & .10 \\
\hline & Total & .41 & .65 & .78 & .63 & .95 & .56 \\
\hline & Overall Total & .29 & .47 & .61 & .52 & .84 & .42 \\
\hline
\end{tabular}

relations. For implicit problem relations, however, the superiority of the matrix mode of representation is readily apparent. The proportion of errors on implicit relations was significantly lower for matrix users $(41 \%)$ than for the next lowest category, local grouping users $(65 \%), \mathrm{z}=2.89, \mathrm{p}=.002$.

Within each category of problem relation, errors of omission were three to four times more frequent than errors of commission for both explicit relationships, $\mathrm{z}=$ $2.40, p=.008$, and implicit relationships, $z=7.42$, $p<.001$. The effects of omission errors were also observed when the amount of problem information was increased as the number of values increased from three to five to seven. A sharp rise in the mean proportion of these errors for implicit relations was observed both for problems of five dimensions $(33 \%, 62 \%$, and $76 \%)$ and for problems of seven dimensions $(28 \%, 53 \%$, and $82 \%)$, but not for problems with only three dimensions (23\%, $28 \%$, and $23 \%$ ). This resulted in a significant values effect, $F(2,171)=15.84, \mathrm{p}<.001$, as well as a significant interaction between dimensions and values, $\mathrm{F}(4,171)=3.45, \mathrm{p}<.01$, with $\mathrm{SE}=.08$ for both of these analyses. Omission errors for explicit problem relations, however, as well as commission errors for both types of relationships, produced comparatively little influence on performance. Hence, the overall difficulty of these problems is best reflected in the pattern of omission errors of implicit relations that-must be deduced from explicitly presented information.

\section{DISCUSSION}

Naive Ss appear only moderately sensitive to the increasing advantage of a matrix mode of representation as the size of the who-done-it problem increases. This occurs despite the fact that a matrix format seems almost a necessity for success in 5 by 7,7 by 5 , and 7 by 7 problems. The most likely explanations for this are either that many Ss are not familiar with matrices or cannot break their set or restructure their field (Wertheimer, 1945) even in the face of an inadequate, unsuccessful representation. Previous studies confirm the general tendency to remain with the same representation throughout a series of problems (Schwartz, 1971; Schwartz \& Fattellah, 1972).
As in the above studies, the overall success rate for those Ss who did use a matrix representation is significantly higher than for Ss using other modes. The analysis of specific types of errors committed in these problems suggests that the locus of effect is not in providing a representation into which it is easier to encode information. Rather, it aids in generating a solution space where deductive transformations, syntheses, and storage of results may be more readily carried out. Precisely why a matrix is so optimal for execution and storage is still an open question for which there is little conclusive evidence as yet.

The general lack of success with the three largest problems, even for Ss using a matrix representation (5\% to $10 \%$ solution rates), suggests that these problems may have exceeded the limit of some basic information processing capacity in many Ss (all Ss worked enthusiastically so that lack of motivation did not appear to be a factor). It is only these three problems that require Ss on occasion to consider and test five to seven possible contingencies in order to deduce a required relationship. A less glamorous but plausible hypothesis involves the dramatic increase in blind alleys (fruitless starting points) after the very beginning of these problems (e.g., an average of 16.5 starting points for the 7 by 5 and 7 by 7 problems, compared to an average of 8.5 for the 5 by 5 and 5 by 7 problems). Future research will hopefully present more evidence on the interaction of the modes of representation into which we encode information presented in problems and the limitations in further processing and retrieval of such information.

\section{REFERENCES}

Greeno, J. G. The structure of memory and the process of solving problems. In R. L. Solso (Ed.), Contemporary issues in cognition. New York: Wiley, 1973 .

Newell, A., Shaw, J. C., \& Simon, H. A. Elements of a theory of human problem solving. Psychological Review, 1958, 65, 151-166.

Schwartz, S. H. Modes of representation and problem solving: Well evolved is half solved. Journal of Experimental Psychology, 1971, 91, 347-350.

Schwartz, S. H., \& Fattellah, D. Representation in deductive problem solving. Journal of Experimental Psychology, 1972, 95, 343-348.

Wertheimer, M. Productive thinking. New York: Harper, 1945. 